Check for updates

Cite this: J. Mater. Chem. A, 2021, 9 , 24631

Received 20th April 2021

Accepted 14th October 2021

DOI: $10.1039 / \mathrm{d} 1 \mathrm{ta03307g}$

rsc.li/materials-a

\section{Crystalline boron monosulfide nanosheets with tunable bandgaps $\dagger$}

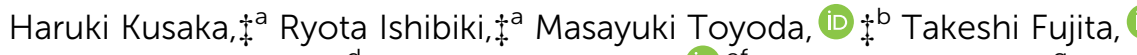
Tomoharu Tokunaga, ${ }^{d}$ Akiyasu Yamamoto, (D) ef Masashi Miyakawa, ${ }^{9}$ Kyosuke Matsushita, ${ }^{\text {h }}$ Keisuke Miyazaki, ${ }^{i}$ Linghui Li, ${ }^{a}$ Satish Laxman Shinde, ${ }^{j}$ Mariana S. L. Lima, (D) ${ }^{a}$ Takeaki Sakurai, (D) ${ }^{\mathrm{kl}}$ Eiji Nishibori, (D) ${ }^{\mathrm{m}}$ Takuya Masuda, ${ }^{\mathrm{h}}$ Koji Horiba, ${ }^{\mathrm{n}}$ Kenji Watanabe, (D) ${ }^{9}$ Susumu Saito, ${ }^{\text {bfo }}$ Masahiro Miyauchi, (D) ${ }^{\text {i Takashi Taniguchi, }}{ }^{p}$ Hideo Hosono (D) fp and Takahiro Kondo (D)*fil

Two-dimensional (2D) boron monosulfide (BS) nanosheets are predicted to have several stable phases and unique electronic structures, endowing them with interesting attributes, including superconducting, thermoelectric, and hydrogen storage properties. In this paper, we report the experimental realization of 2D BS nanosheets by the physical exfoliation of rhombohedral boron monosulfide ( $r$-BS). Moreover, we demonstrate the facile separation of a mixture of 2D BS nanosheets and the r-BS powder in acetonitrile; the former were selectively separated as a dispersion in the supernatant, whereas the latter remained in the precipitate. In addition, density functional theory calculations reveal a clear dependence of the bandgap energy $\left(E_{\mathrm{g}}\right)$ on the number of layers of stacked BS nanosheets, where $E_{\mathrm{g}}$ for BS nanosheets is approximately $1.0 \mathrm{eV}$ higher than that for $\mathrm{r}$-BS. Atomic force microscopy, cathode luminescence, ultraviolet-visible absorption spectroscopy, and excitation emission matrix experiments revealed a consistent bandgap difference of approximately $1.0 \mathrm{eV}$ between the BS nanosheets and $\mathrm{r}$-BS. We also demonstrate the applications based on the properties that originated from the difference in the bandgap between $r$-BS and BS nanosheets using photoelectrochemical current switching. These results indicate that the nanosheet bandgap can be tuned to a desired value by controlling the number of stacked 2D BS nanosheets. Therefore, BS nanosheets are promising non-metal 2D materials for applications requiring bandgap control, such as electronics and photocatalysis.
${ }^{a}$ Graduate School of Pure and Applied Sciences, University of Tsukuba, Tsukuba 305-8573, Japan

${ }^{b}$ Department of Physics, Tokyo Institute of Technology, Meguro-ku, Tokyo 152-8551, Japan

'School of Environmental Science and Engineering, Kochi University of Technology, Kochi 782-8502, Japan

${ }^{d}$ Graduate School of Engineering Materials Design Innovation Engineering, Nagoya University, Aichi 464-8601, Japan

'Institute of Engineering, Tokyo University of Agriculture and Technology, Tokyo 1838538, Japan

${ }^{f}$ Materials Research Centre for Element Strategy, Tokyo Institute of Technology, Yokohama 226-8503, Japan

${ }^{g}$ Research Center for Functional Materials, National Institute for Materials Science, Tsukuba 305-0044, Japan

${ }^{h}$ Center for Green Research on Energy and Environmental Materials, National Institute for Materials Science, Tsukuba 305-0044, Japan

${ }^{i}$ Department of Materials Science and Engineering, School of Materials and Chemical Technology, Tokyo Institute of Technology, Meguro-ku, Tokyo 152-8552, Japan
${ }^{j}$ Department of Materials Science, Faculty of Pure and Applied Sciences, University of Tsukuba, Tsukuba 305-8573, Japan

${ }^{k}$ Department of Applied Science, Faculty of Pure and Applied Sciences, University of Tsukuba, Tsukuba 305-8573, Japan

${ }^{\prime}$ Tsukuba Research Center for Energy Materials Science, Faculty of Pure and Applied Sciences, University of Tsukuba, Tsukuba 305-8573, Japan

${ }^{m}$ Department of Physics, Faculty of Pure and Applied Sciences, University of Tsukuba, Tsukuba 305-8571, Japan

${ }^{n}$ Condensed Matter Research Center and Photon Factory, Institute of Materials Structure Science, High Energy Accelerator Research Organization (KEK), Tsukuba 305-0801, Japan

${ }^{\circ}$ Advanced Research Center for Quantum Physics and Nanoscience, Tokyo Institute of Technology, Meguro-ku, Tokyo 152-8551, Japan

${ }^{p}$ International Center for Materials Nanoarchitectonics, National Institute for Materials Science, Tsukuba 305-0044, Japan

$\dagger$ Electronic supplementary information (ESI) available. See DOI: $10.1039 / \mathrm{d} 1 \mathrm{ta} 03307 \mathrm{~g}$

\$ These authors contributed equally. 


\section{Introduction}

Two-dimensional (2D) materials have unique properties, including large surface areas and novel electronic states, ${ }^{1-3}$ endowing them with great potential for many applications, with batteries, catalysts, and electronic devices as examples. In addition, combining 2D materials ${ }^{4}$ or $2 \mathrm{D}$ and otherdimensional materials ${ }^{5}$ can result in new physical properties. Intriguingly, unconventional properties, including superconductivity, have been reported in 2D superlattices created by stacking two sheets of 2D materials and twisting them relative to each other by a small angle. ${ }^{6}$ Hence, 2D materials are potential building blocks for creating new materials with desirable and controllable functionalities. Among these 2D materials, boron-related materials are of particular interest because of their polymorphism ${ }^{7-9}$ and ability to easily form multi-center bonds. ${ }^{10}$ Theoretically, boron exhibits a wide variety of stable 2D phases, including borophene, ${ }^{7,11-13}$ hydrogenated 2D boron (borophane or hydrogen boride), ${ }^{9}$ 2D boron monosulfide (BS), ${ }^{14}$ 2D boron oxide, ${ }^{15}$ and 2D boron phosphide. ${ }^{16}$ Hence, several building blocks can be obtained by tuning the bonding configurations of $2 \mathrm{D}$ boron nanomaterials. However, to date, only borophene, ${ }^{17-19}$ borophane, ${ }^{20-23}$ boric acid, and boron nitride have been realized experimentally, and, to the best of our knowledge, no other 2D boron compounds have been reported. Nevertheless, a recent theoretical study predicted that $2 \mathrm{D}$ BS exists in several stable phases with unique electronic structures, ${ }^{24}$ including superconducting, ${ }^{14}$ thermoelectric, ${ }^{25}$ and hydrogen storage properties. ${ }^{26}$

In this paper, we report the preparation of 2D BS nanosheets by physically exfoliating bulk rhombohedral boron monosulfide (r-BS). Because the synthesis of r-BS has only been reported by a few groups, ${ }^{27-29}$ we carefully characterized r-BS, including its thermal stability. Further, after exfoliating r-BS to form 2D BS nanosheets, we found that the $2 \mathrm{D}$ BS nanosheets and r-BS exhibit distinctly different bandgaps, and this bandgap difference was reproduced using density functional theory (DFT) calculations. The calculation further revealed that the bandgap energy depends on the stacking number of BS nanosheets. Based on these findings, 2D BS nanosheets are potential bandgap-tunable materials for advanced applications.

\section{Results and discussion}

The parent material, r-BS, was synthesized following previous reports of its synthesis. ${ }^{27-29}$ Briefly, a mixture of sulfur and amorphous boron in a $1: 1$ atomic ratio was heated to $1873 \mathrm{~K}$ at $5.5 \mathrm{GPa}$ and then quenched to room temperature (approximately $300 \mathrm{~K}$ ). The obtained product pellet was easily crushed into a powder. The synchrotron radiation X-ray diffraction (SRXRD) pattern of the powder and its Rietveld analysis shown in Fig. 1a clearly indicates that r-BS has a trigonal structure with $R \overline{3} m$ symmetry and was formed as a single phase (above $99.2 \%$ purity of phase) under the experimental conditions (acquired crystal data are listed in Table $\mathrm{S} 1, \uparrow$ see Experimental section for details). The determined B-B distance of 1.6756(1) corresponds to that of a B-B single bond $(1.72 \AA),{ }^{30}$ rather than a double $(1.560 \AA)^{31}$ or triple bond (1.455-1.504 $\left.\mathrm{\AA}\right) .^{32}$ X-ray photoelectron spectroscopy (XPS) of r-BS yielded singlecomponent $\mathrm{B} 1 \mathrm{~s}$ and $\mathrm{S} 2 \mathrm{p}_{3 / 2}$ (merged with $\mathrm{S} 2 \mathrm{p}_{1 / 2}$ ) spectra, as shown in Fig. 1b. The binding energies of these peaks indicate that the charge states of B and S are close to neutral (Fig. S1 $\dagger$ ), whereas sulfur is slightly negatively charged, which is ascribable to electron accumulation in $\mathrm{p}_{z}$ orbital of sulfur based on the density functional theory (DFT) calculation (Fig. S2 $\dagger$ ). Our DFT results with the electron localization function (ELF) ${ }^{33,34}$ analysis also show that maximum electron density is located at the midpoint between B and S of BS bond, which is sharp contrast to the case of metal sulfides such as $\mathrm{MoS}_{2}$ (Fig. S2 $\dagger$ ). Thus, we conclude that the $\mathrm{B}$ and $\mathrm{S}$ atoms in r-BS are covalently bonded. Based on the areas of the XPS B 1s and S 2p (or S 2s) peaks and their sensitivity factors (B: 2.1017, S 2p3/2: 7.1859, S 2s: 6.1445$)$, the B : $\mathrm{S}$ atomic ratio was calculated to be $1 \pm 0.1: 1$ \pm 0.1 , indicating that sulfur was not lost from the sample cell during high-pressure synthesis, even at a temperature of 1873 $\mathrm{K}$, and all boron and sulfur detected at the surfaces of the powder particles are present as r-BS. As shown in Fig. 1c, the Raman spectra show three distinct peaks at 319, 687, and $1041 \mathrm{~cm}^{-1}$, which are ascribed to the $\mathrm{A}_{1}(3), \mathrm{E}(4)$, and $\mathrm{A}_{1}(4)$ modes of r-BS, respectively. ${ }^{29}$ The absence of other peaks corresponding to boron or sulfur compounds in the Raman spectra indicates that r-BS had been synthesized as a single phase, consistent with the SRXRD data. The scanning electron microscopy (SEM) images (Fig. 1d and S3†) show that the r-BS particles are mostly larger than $10 \mu \mathrm{m}$, but some particles are a few hundred nanometers in size. The observed particles were found to be composed of boron and sulfur, as shown in Fig. 1d, based on the elemental maps obtained using an electron probe microanalyzer (EPMA). The same trend was also observed by energy dispersive X-ray spectroscopy (EDS) (Fig. S4†). To evaluate its thermal stability, r-BS was subjected to thermogravimetric analysis (TGA) under a flow of Ar, the results of which are shown in Fig. 1e. The weight of the r-BS sample did not change up to $700 \mathrm{~K}$, which indicates thermal stability up to this temperature. Further, the XRD pattern of the sample heated at $725 \mathrm{~K}$ under a flow of Ar was consistent with that of the original phase-pure r-BS (Fig. S5†). However, gradual weight loss was observed once the temperature reached approximately $730 \mathrm{~K}$, and the XRD pattern was also different after heating to $1000 \mathrm{~K}$ in $\mathrm{Ar}$, which indicates that r-BS is thermally unstable above $730 \mathrm{~K}$.

Transmission electron microscopy (TEM) revealed that the $\mathrm{r}$ BS powder sample contained thin layers (2D sheets). The images in Fig. 2a show some folded regions that are characteristic of $2 \mathrm{D}$ sheets. A clear electron diffraction pattern was obtained from the same location (see inset in Fig. 2a), which indicates that the $2 \mathrm{D}$ sheets are crystalline. In addition, distinct boron and sulfur signals were detected by electron energy loss spectroscopy (EELS) at this position, as shown in Fig. 2b, which reveals that the crystalline $2 \mathrm{D}$ sheets are composed of boron and sulfur. High-resolution TEM was used to observe the real-space structure of this layer directly at atomic resolution, the results of which are shown in Fig. 2c. The periodic bright and dark spots confirm that the 2D sheets are crystalline. Further, the profile 


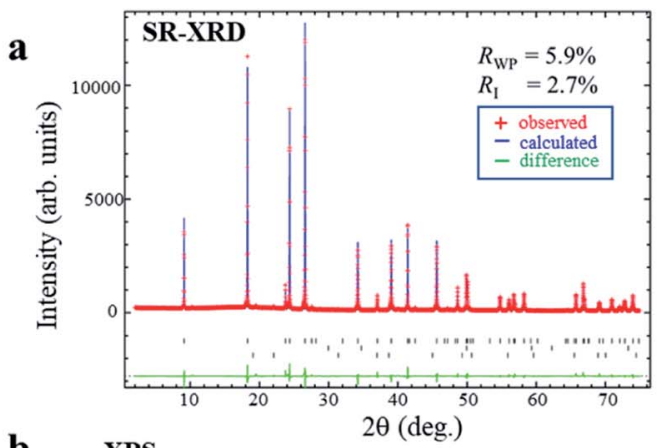

b
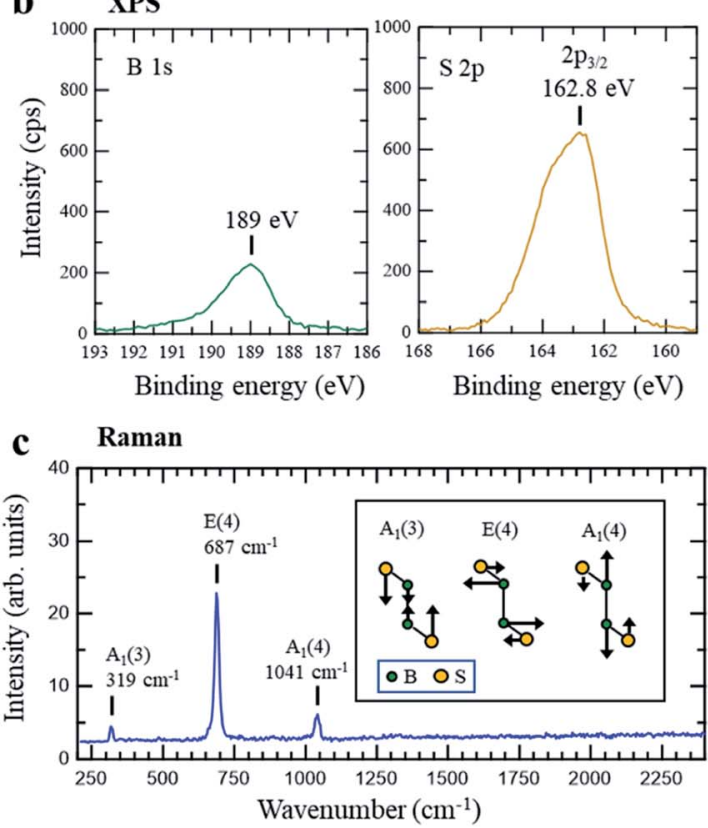

d
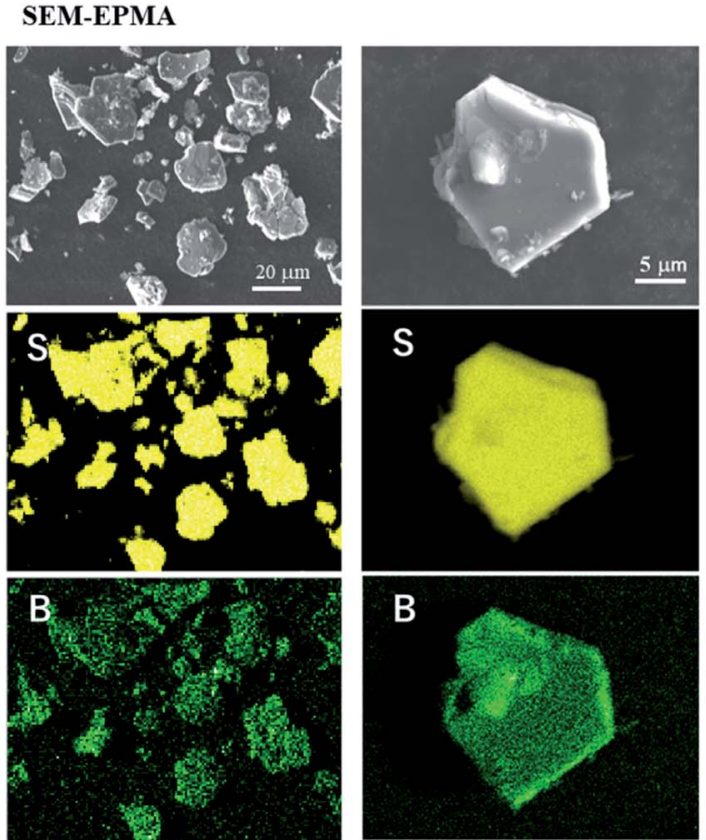

e

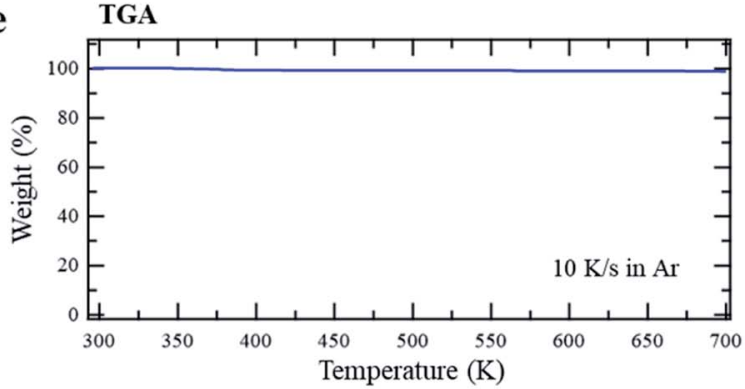

Fig. 1 (a) Synchrotron radiation X-ray diffraction (SRXRD) pattern of $r$-BS powder with Rietveld analysis. (b) X-ray photoelectron spectra of $r$-BS powder on a Au surface. (c) Raman spectroscopy of $r$-BS powder (inset image shows the vibrational modes of $r$-BS ${ }^{29}$ ). (d) Scanning electron microscopy (SEM) images and sulfur and boron elemental maps of $r$-BS powder particles on a Si surface obtained using an electron probe micro analyzer (EPMA). The incident energy of the electron beam was $15 \mathrm{kV}$. (e) Thermogravimetric analysis (TGA) of $r$-BS powder heated at $10 \mathrm{~K} \mathrm{~s}^{-1}$ under a flow of Ar.

along the line between A-A' in Fig. 2c (see Fig. 2d) contains 57 peaks over a distance of $10.42 \mathrm{~nm}$; thus, the average peak-topeak distance is $0.18 \pm 0.1 \mathrm{~nm}$, which is consistent with the sulfur-sulfur distance of the (001) surface of r-BS (distance between the ends of the red arrows in the top-view of r-BS shown in Fig. 3a). These results indicate that the as-prepared r-BS sample powder contains 2D BS nanosheets. We believe that these 2D BS nanosheets were formed by exfoliation as the pellets were crushed into the powder. Indeed, the obtained powder is composed of large (micrometer-sized) particles and small (about $100 \mathrm{~nm}$ ) particles (Fig. 1d and S3†), which is consistent with the theoretical prediction that r-BS is easily exfoliated to form nanosheets. ${ }^{\mathbf{1 4}}$ Crucially, our TEM observations suggest that the 2D BS nanosheets formed by the exfoliation of r-BS preserve the original crystal structure.

To explore the differences in the physical properties of r-BS and the 2D BS nanosheets, we calculated the band structure as a function of the number of stacked BS nanosheets using DFT calculation. Here, the structure of the monolayer BS and the way of stacking are assumed to be the same as those of the three layers of r-BS (trigonal structure with $R \overline{3} m$ symmetry), as shown schematically in Fig. 3a. Geometries were optimized from this starting structure and band structures were calculated. The calculated band structures of r-BS and monolayer BS are shown in Fig. 3b and c, respectively. The effective electron mass of monolayer BS (average mass $m_{\mathrm{e}}^{*}=0.29$ ) is less than that of r-BS $\left(m_{\mathrm{e}}^{*}=0.41\right)$ (Table $\left.\mathrm{S} 2 \dagger\right)$ owing to the parabolic dispersion along the $\mathrm{M}-\mathrm{K}$ line (Fig. 3c), whereas the effective hole mass of monolayer BS (average mass $m_{\mathrm{h}}^{*}=6.95$ ) is more than that of $\mathrm{r}$-BS $\left(m_{\mathrm{h}}^{*}=0.57\right)$. These results suggest the potential application of monolayer BS as an n-type semiconductor with high conductivity. This is a unique property among boronbased nanomaterials and sharp contrast to borophene and borophane, which do not have a bandgap. Owing to the overlap of the outermost sulfur orbitals, the valence band top state (composed of the hybridized $\mathrm{p}_{z}$ orbitals of boron and sulfur) of r-BS shifts to higher energy compared to that of the BS monolayer through bonding/anti-bonding splitting. Thus, the 
a

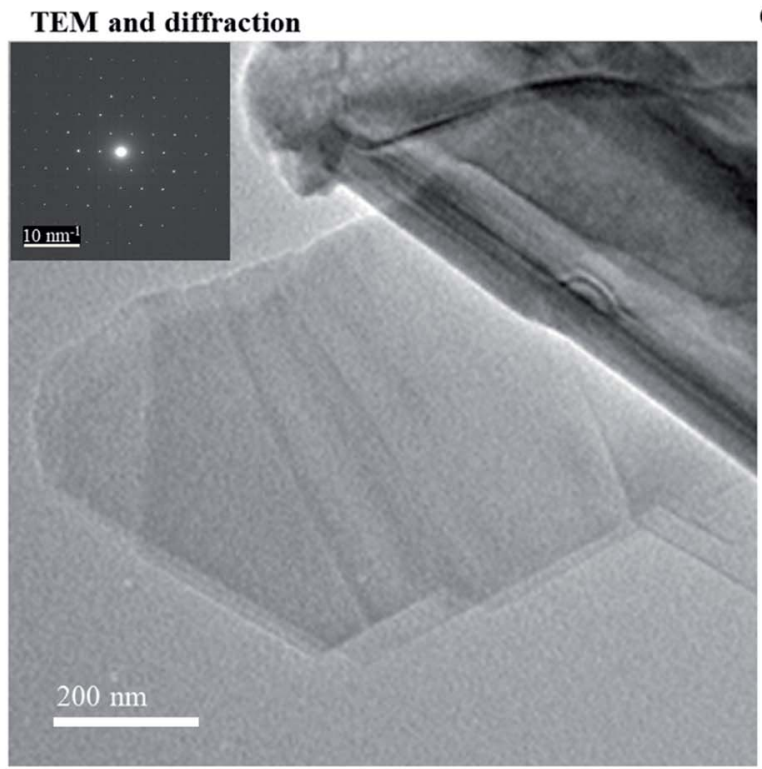

c TEM

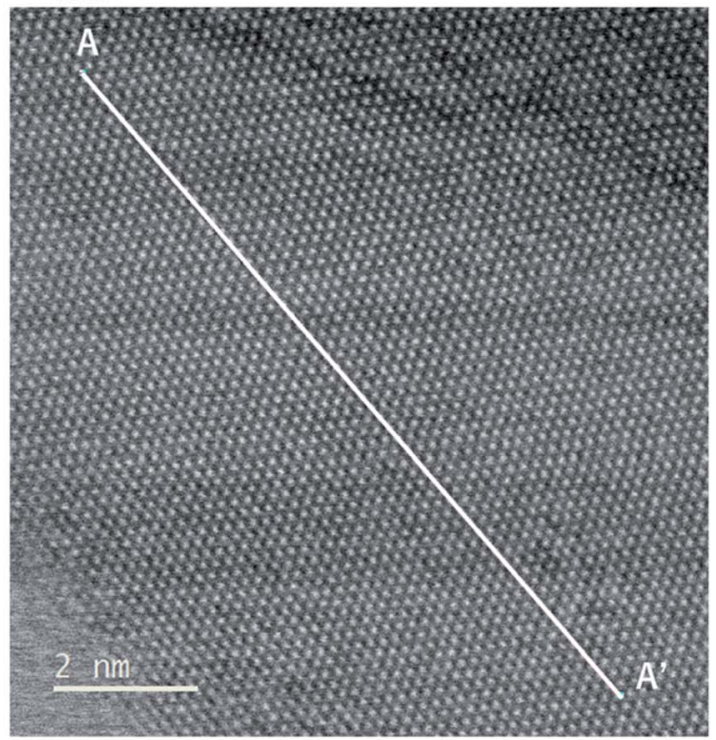

b

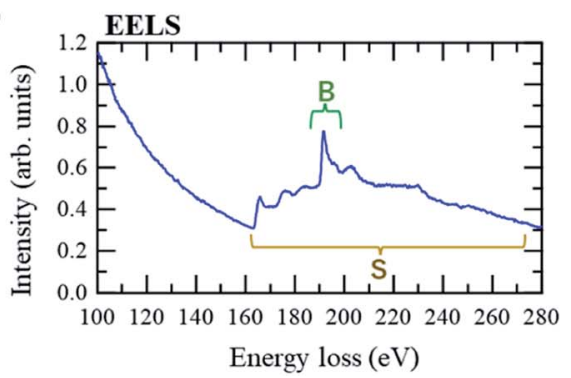

d

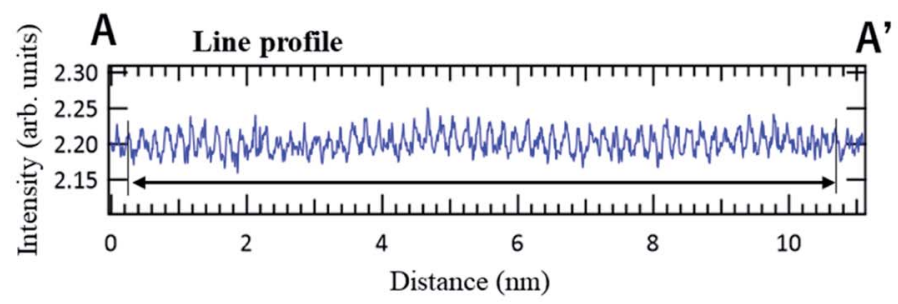

Fig. 2 (a) Transmission electron microscopy (TEM) image of a thin layer (2D BS nanosheets) in the r-BS powder, as well as an electron diffraction pattern obtained for the same thin layer. (b) Electron energy loss spectroscopy (EELS) results for $r$-BS powder. (c) High-resolution TEM image of the thin layer observed in panel a. (d) Intensity profile along the line between points A and $A^{\prime}$ in the TEM image in panel $\mathrm{C}$.

bandgap of r-BS $2.7 \mathrm{eV}$ using the Heyd-Scuseria-Ernzerhof hybrid functional method, HSE06, and $1.7 \mathrm{eV}$ using local density approximation, LDA) is smaller than that of monolayer BS (3.6 eV using HSE06 and $2.9 \mathrm{eV}$ using LDA). In every case, an indirect (rather than direct) bandgap was identified, which can be changed to a direct bandgap by applying biaxial mechanical strain, as suggested for transition-metal chalcogenides. ${ }^{35}$ The calculated bandgap of r-BS is smaller than the experimentally estimated value $(3.4 \mathrm{eV})$ using ultraviolet-visible (UV-Vis) absorption spectroscopy. ${ }^{27}$ The dependence of the bandgap on the number of BS layers is plotted in Fig. 3d. In particular, the bandgap energy decreased notably as the stacked BS layers were increased from one to three, but gradually decreased to the bulk r-BS bandgap value. The difference between bandgap of monolayer BS and bulk r-BS is approximately $1.0 \mathrm{eV}$, and the difference is consistent for both HSE06 and LDA methods. This result reveals that the desired bandgap can be obtained by tuning the number of stacked 2D BS nanosheets, as has been reported for 2D transition metal dichalcogenide (TMD; e.g., $\left.\mathrm{MoS}_{2}\right)^{36}$ and quaternary 2D TMD nanosheets. ${ }^{37}$ Hence, the BS nanosheet is a new addition to the family of promising $2 \mathrm{D}$ materials as non-metal materials for applications requiring bandgap control, such as electronics and photovoltaics.
To verify the predicted bandgap difference between r-BS and the 2D BS nanosheets experimentally, we prepared 2D BS nanosheets by physical (mechanical) exfoliation, using the wellknown Scotch-tape method. ${ }^{38}$ Specifically, the r-BS powder was placed on sticky tape, and the tape was folded and separated several times to create progressively thinner layers of BS, after which, the tape was placed in contact with a mica surface to transfer the BS layers onto the mica. Fig. $4 \mathrm{a}$ and b show atomic force microscopy (AFM) images of r-BS before and after exfoliation. As shown in the line profiles of the AFM images (Fig. 4c and d), the observed heights of the sheets on mica were approximately 130 and $1 \mathrm{~nm}$, respectively, indicating the presence of a thin film on the mica surface comprising a few layers of 2D BS sheets after transfer of the exfoliated sheets. Fig. 4e and $f$ show cathodoluminescence (CL) spectra of the r-BS and 2D BS nanosheets prepared on the Si surface (spectra obtained at different positions are shown together). The main peaks of bulk r-BS are located at 600-700 nm, whereas a distinct peak at $450 \mathrm{~nm}$ and a small peak at $600 \mathrm{~nm}$ were observed for the 2D BS nanosheets. A comparison of the peak positions with the bandgaps predicted by DFT (Fig. 3d) shows that the peaks at 600-700 and $450 \mathrm{~nm}$ are ascribable to luminescence from thick r-BS stacks and those having one or two BS layers, respectively. A 
a
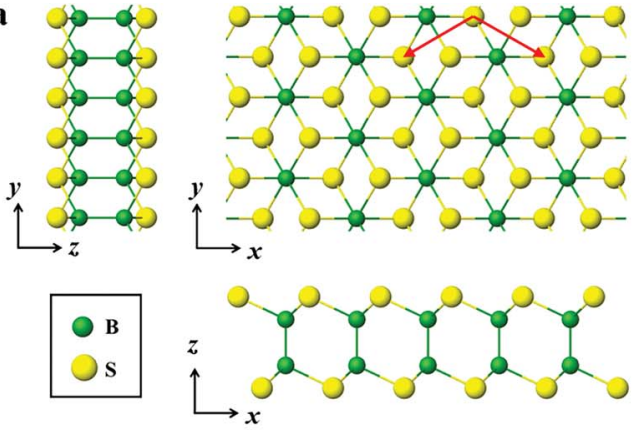

Bulk (r-BS)

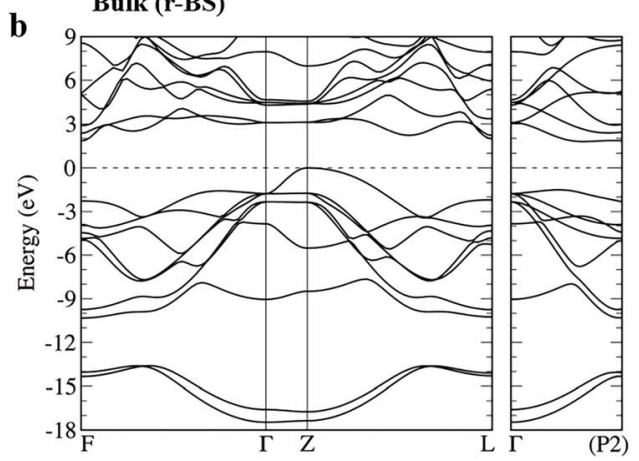

Monolayer BS

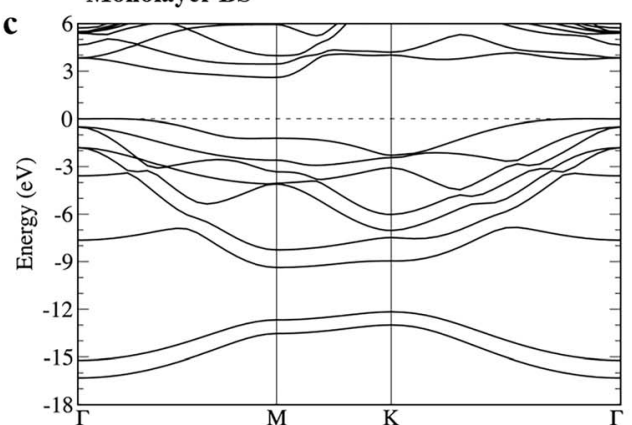

d

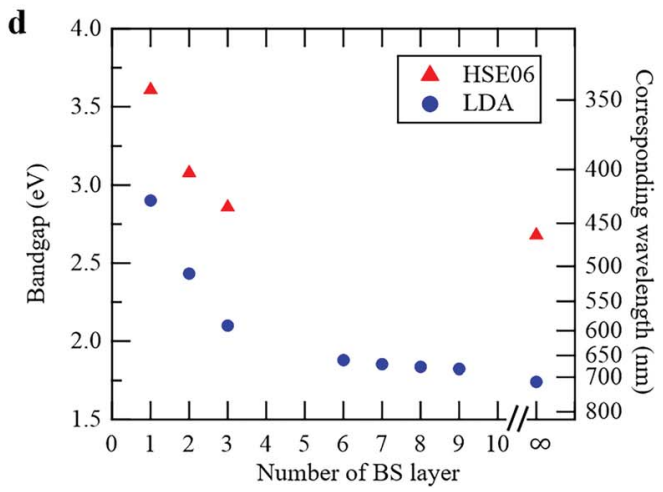

Fig. 3 (a) Schematic showing 2D BS nanosheets. The crystal structure figures of the 2D BS nanosheet were generated using VESTA. ${ }^{50}$ (b) DFT-LDA band structure of $r$-BS. (c) DFT-LDA band structure of monolayer BS. (d) DFT-calculated bandgap using HSE06 (red triangle) and LDA (blue circle) of $r$-BS as a function of the number of stacked BS layers.

similar bandgap difference was observed during the UV-Vis absorption spectroscopy (Fig. S6 $\dagger$ ). Although a previous report attributed the visible light absorption of r-BS bulk to the defects, ${ }^{27}$ our DFT calculation indicates that the visible light absorption of the bulk r-BS can be attributed to its intrinsic property. The spectrum of r-BS was measured using a powder sample, while that of BS nanosheets was obtained using dispersion in acetonitrile; therefore, the bandgap values could not be distinguished quantitatively. The difference in the experimental conditions yields different backgrounds for the UV-Vis spectra in Tauc-plot analysis, hindering the experimental calculation of bandgap from the UV-Vis spectra. In any case, our AFM and CL data clearly reveal that the structural properties and luminescence behavior of r-BS and the 2D BS nanosheets are quite different (by as much as $1 \mathrm{eV}$ ), and this difference is ascribable to the difference in the bandgaps of r-BS and the BS nanosheets revealed by the DFT calculations. Additionally, the excellent environmental stability of BS nanosheets prepared using the Scotch-tape method was confirmed by examining the surface states using XPS of the sample after exposure to air and after drying the distilled water droplet (Fig. S7†).

Next, we demonstrated the facile separation of the synthesized mixture of 2D BS nanosheets and r-BS powder in acetonitrile. Specifically, the 2D BS nanosheets were selectively separated as a dispersion in the supernatant, whereas the r-BS remained in the precipitate. Fig. 5 shows the excitation-emission matrices (EEMs) of the precipitate and supernatant of the as-synthesized r-BS powder in acetonitrile. A distinct difference in the emission peaks is evident; specifically, after separation in acetonitrile, an EEM peak appears at 500-700 $\mathrm{nm}$ for the precipitate, whereas a peak at $400-450 \mathrm{~nm}$ was observed for the supernatant. These peaks are ascribed to r-BS and 2D BS nanosheets, respectively, based on the CL and DFT results (Fig. 3d and 4). XPS, AFM, and Raman mapping were used to confirm these assignments for both samples, which indicated that the dispersion in the supernatant and the precipitate obtained in acetonitrile indeed correspond to r-BS and the 2D BS nanosheets, respectively (Fig. S8-S11†).

Finally, an application based on the property originating from the difference in the bandgap between r-BS and BS nanosheets is demonstrated. The results of photoelectrochemical property measurements are shown in Fig. 6. The electrode of r-BS was responsive to visible light over $422 \mathrm{~nm}$ (Fig. 6a), whereas that of 2D BS nanosheet was responsive only under ultraviolet (UV) light (Fig. 6b). These trends are consistent with their optical fluorescence properties. The hydrogen evolution reaction proceeds by light irradiation owing to the absence of added redox agent. The photocatalytic properties of these electrodes will be comprehensively studied and reported in the future. Based on the present photoelectrochemical results, the dependence of wavelength on photocurrent can be controlled using sheet or bulk form of BS. Therefore, the r-BS can be used for developing sensors and photovoltaic devices.

\section{Conclusions}

We prepared 2D BS sheets using the physical exfoliation of r-BS. Our XRD, XPS, SEM, EPMA, and Raman scattering experiments show that single-phase crystalline r-BS with trigonal structure 
a

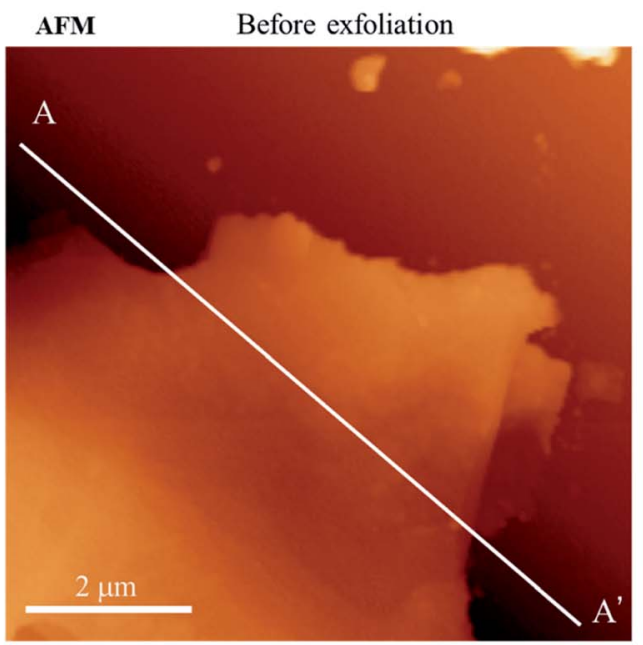

c

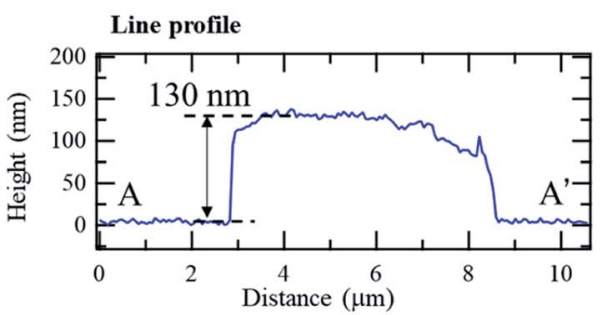

e

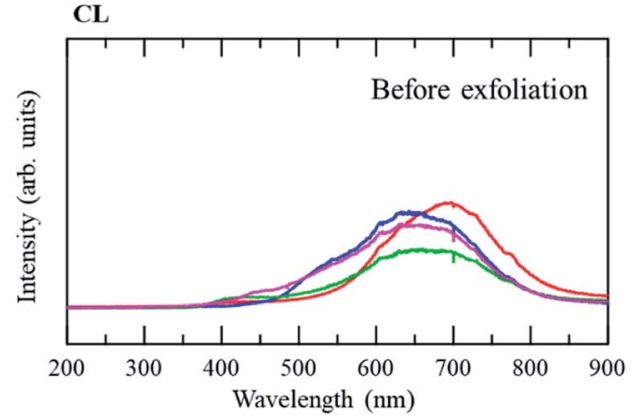

b

b AFM

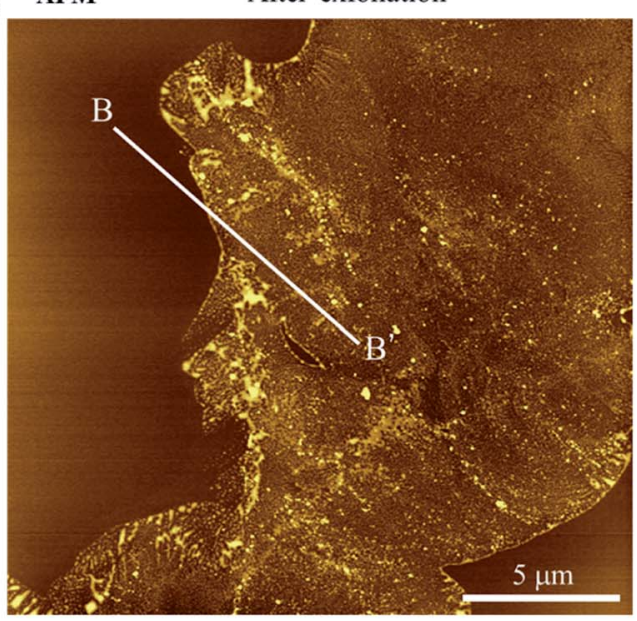

d

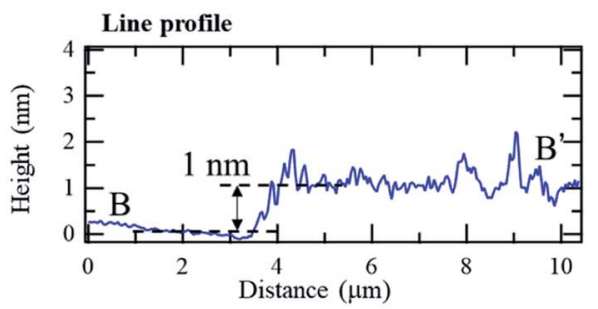

CL

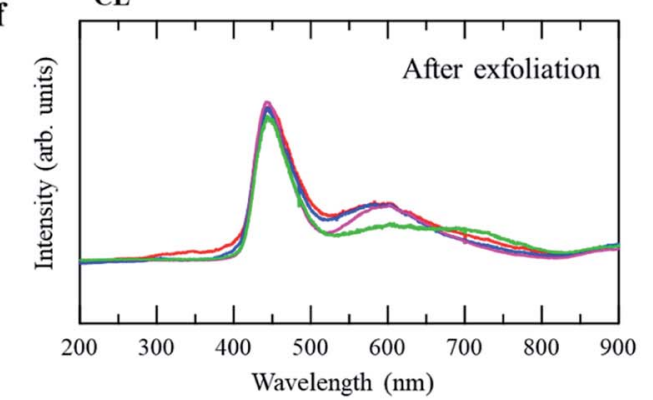

Fig. 4 (a) Atomic force microscopy (AFM) image of $r$-BS powder (before exfoliation) on a mica surface. (b) AFM image of the 2D BS nanosheets (after the exfoliation of $r$-BS) on a mica surface. (c) Height profile obtained along the line between points A and $A^{\prime}$ in the AFM image in panel a. (d) Height profile obtained along the line between points $B$ and $B^{\prime}$ in the AFM image in panel $b$. (e) Cathodoluminescence (CL) spectra obtained at different positions of the $r$-BS powder on a Si surface. (f) CL spectra of 2D BS nanosheets (after the exfoliation of $r$-BS) on a Si surface.

and $R \overline{3} m$ symmetry can be synthesized by heating a mixture of boron and sulfur (B:S=1:1) to $1873 \mathrm{~K}$ at $5.5 \mathrm{GPa}$, followed by quenching to room temperature (approximately $300 \mathrm{~K}$ ). TGA in Ar and the XRD pattern showed that r-BS is thermally stable up to $730 \mathrm{~K}$. TEM, EELS, and electron diffraction experiments show that 2D BS nanosheets are contained in the synthesized r-BS powder, possibly due to exfoliation during the crushing of the pellet into powder. The observed 2D BS nanosheets are likely to have crystalline structures that correspond to the same layer structure as r-BS; that is, the 2D BS nanosheets were formed by the exfoliation of r-BS, thereby preserving the original crystalline structure. DFT calculations show a clear dependence of the bandgap energy $\left(E_{\mathrm{g}}\right)$ on the number of stacked BS layers, where $E_{\mathrm{g}}$ for BS nanosheets is approximately $1.0 \mathrm{eV}$ higher than that for r-BS. In addition, AFM, CL, UV-Vis, and EEM experiments reveal that there are corresponding bandgap differences (as much as $1 \mathrm{eV}$ ) between r-BS and the 2D BS nanosheets (prepared by the Scotch-tape method). Moreover, the mixture of r-BS and 2D BS nanosheets was found to be easily separated into r-BS and 2D BS nanosheets in acetonitrile as a dispersion in the supernatant and as a precipitate, respectively. Finally, we demonstrated an application based on the property that originated from the difference in the bandgap between r-BS and BS nanosheets by photoelectrochemical current switching.

\section{Experimental}

\section{r-BS synthesis}

Amorphous boron (99.9\%) was prepared by the decomposition of $\mathrm{B}_{2} \mathrm{H}_{6}$. A mixture of amorphous boron (214.3 mg) and sulfur (635.7 mg, 99\%, Wako Pure Chemical Industries Ltd., Osaka, Japan) was stirred in an agate mortar for $15 \mathrm{~min}$. The mixture was then converted to a pellet by pressing at $150 \mathrm{kgf} \mathrm{cm}^{-2}$. The pellet was then placed in a boron nitride (BN) capsule, which 

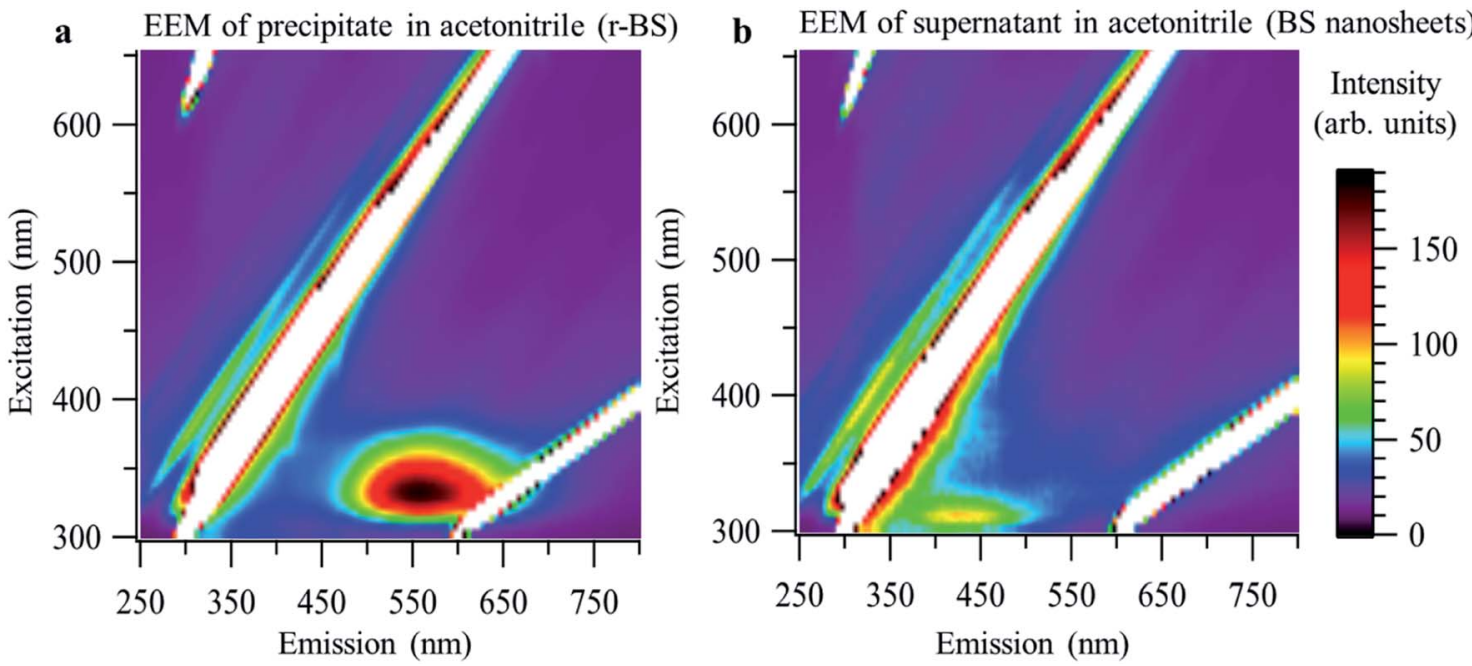

Fig. 5 Excitation-emission matrices (EEMs) of $r-B S$ and 2D BS nanosheets. (a) EEM of the precipitate in acetonitrile ( $r$-BS). (b) EEM of the 2D BS nanosheets (supernatant in acetonitrile).

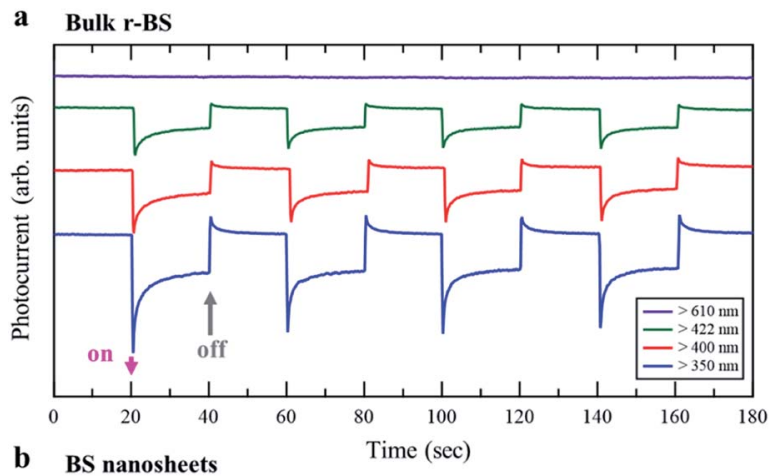

b

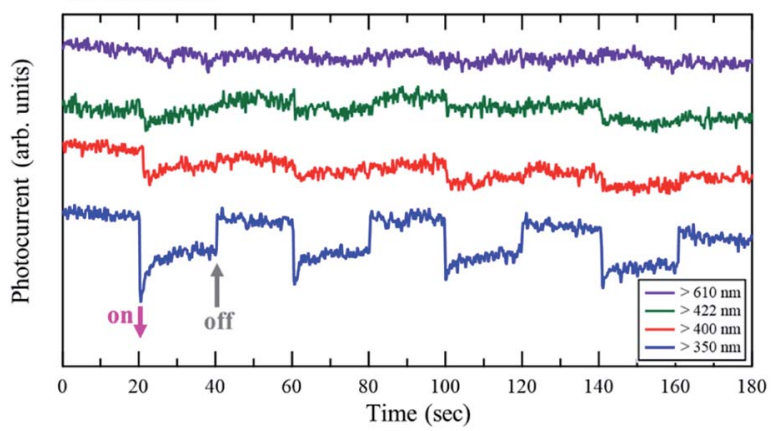

Fig. 6 Photoelectrochemical current response under light on and off conditions (repeated every 20 seconds), (a) for bulk $r$-BS, and (b) for BS nanosheets. Light was irradiated using a xenon lamp passed through a short wavelength cut-off filter below 610, 422, 400, or $350 \mathrm{~nm}$.

was then sandwiched by $\mathrm{NaCl}$ disks and then sandwiched by graphite disks. The pressure was first increased up to $5.5 \mathrm{GPa}$, and, then, the temperature was increased to $1873 \mathrm{~K}$ over $17 \mathrm{~min}$ in a modified belt-type high-pressure apparatus with a cylinderbore diameter of approximately $32 \mathrm{~mm} .{ }^{39}$ After $30 \mathrm{~min}$, the temperature was reduced suddenly (quenched) to room temperature (approximately $300 \mathrm{~K}$ ). The obtained pellet was then carefully scratched to remove the surrounding materials and the pellet inside was crushed to a powder.

\section{XRD}

$\mathrm{XRD}$ patterns were acquired at room temperature (approximately $300 \mathrm{~K}$ ) using a benchtop X-ray diffractometer (Rigaku MiniFlex, Tokyo, Japan) with $\mathrm{Cu} \mathrm{K} \alpha$ radiation. X-rays were generated using the line focus principle. A reflection-free $\mathrm{Si}$ plate was used as the sample stage. Diffraction patterns were recorded using a $\mathrm{D} /$ teX Ultra silicon strip detector (Rigaku) at $0.05^{\circ} \mathrm{s}^{-1}$ up to a $2 \theta$ value of $80^{\circ}$.

\section{SRPXRD}

Synchrotron radiation powder diffraction experiments were carried out at the SPring-8 BL02B2 beamline at approximately $300 \mathrm{~K}$ with an imaging plate as the detector. The wavelength of the incident X-ray was 1.0790(1) A, which was calibrated using a $\mathrm{CeO}_{2}$ standard powder sample by the National Institute of Standards and Technology. The data was Rietveld analyzed using a synchrotron powder program. ${ }^{40} \mathrm{NaCl}$ and $\mathrm{BN}$ volumes of less than $0.7 \%$ and $0.07 \%$ were detected in the powder profiles, respectively. Very small amounts of $\mathrm{NaCl}$ and $\mathrm{BN}$ were treated as second and third phases in the refinement. Reliability factors based on weighted profiles, $R_{\mathrm{wp}}$, and Bragg Intensities, $R_{\mathrm{I}}$, were $5.9 \%$ and $2.7 \%$, respectively. Lattice constants determined by refinement are $a=3.05065(1)$ and $c=20.38458(14)$.

\section{XPS}

XPS spectra were acquired at room temperature (approximately $300 \mathrm{~K}$ ) using a JPS 9010 TR (JEOL Ltd., Japan) with an ultrahigh vacuum chamber and an $\mathrm{Al} \mathrm{K} \alpha$ X-ray source (1486.6 eV). The pass energy was $10 \mathrm{eV}$, the energy resolution (estimated from the $\mathrm{Ag} 3 \mathrm{~d}_{5 / 2}$ peak width of a clean $\mathrm{Ag}$ sample) was $0.635 \mathrm{eV}$, and the uncertainty in the binding energy was \pm 0.05 . The sample was placed on the Au surface, and the Au was attached to the 
sample holder by graphite tape. The sample holder was then introduced into the ultrahigh-vacuum chamber for measurement. Before placing the sample on $\mathrm{Au}$, the Au substrate was cleaned by repetitive $\mathrm{Ar}^{+}$ion etching. Shirley background removal was achieved using SpecSurf (version 1.8.3.7, JEOL Ltd., Japan). Owing to the insufficient contact between Au and the sample holder, charge buildup in the sample caused higher binding energy shifts (approximately $0.5 \mathrm{eV}$ ); thus, based on the $\mathrm{Au} 4 \mathrm{f}$ binding energy, we calibrated the amount of charge build up as $84 \mathrm{eV}$.

\section{TEM, EELS, EDS, and electron diffraction}

These experiments were performed at room temperature (approximately $300 \mathrm{~K}$ ) using a JEM-2100F TEM/STEM (JEOL, Ltd., Japan) with double spherical aberration (Cs) correctors (CEOS GmbH, Heidelberg, Germany) to obtain high-contrast images with a point-to-point resolution of $1.4 \AA$. The lens aberration was optimized by evaluating the Zemlin tableau of amorphous carbon. The residual spherical aberration was almost zero (Cs $=-0.8 \pm 1.2 \mu \mathrm{m}$ with $95 \%$ certainty). The acceleration voltage was set to $200 \mathrm{kV}$. Diffraction patterns were recorded with a camera with a charge-coupled device (CCD) (Gatan, Erlangshen). EELS measurements were carried out using a Gatan GIF Tridiem, and EDS spectra were acquired using a JEOL JED-2300T.

\section{SEM and EPMA}

These experiments were performed on a JXA-8530F (JEOL, Ltd., Japan) operated at 10 or $15 \mathrm{kV}$. Elemental maps of boron and sulfur were obtained by wavelength dispersive spectroscopy (WDS) using an intensity of $191.4 \mathrm{~mm}$ from the Ni/C (LDE2H) crystal and at $172.05 \mathrm{~mm}$ from the pentaerythritol (PETH) crystal, respectively, from each sample position. The samples were placed on Si wafers.

\section{SEM and EDX}

These experiments were performed using an SEM-EDX-WDX system (SEM: Hitachi S-3900N, EDX: Oxford X-Max50, WDX: Oxford INCA WAVE500) operating at $10 \mathrm{kV}$. The samples were placed on Si wafers.

\section{Raman scattering}

The experiment was performed using a multichannel Raman imaging system (ST Japan Inc., Japan) with an incident light wavelength of $532 \mathrm{~nm}$.

\section{Raman mapping}

Raman spectroscopy mapping images were obtained at room temperature using a micro-spectroscopy system (Nanofinder 30, Tokyo Instruments) with a YAG laser of $532 \mathrm{~nm}$ wavelength as an excitation light source.

$\mathbf{C L}$

The CL measurement system comprises an SEM system (Topcon, SM350) and an optically coupled spectrograph system
(Photon Design, PDP-320). This spectrograph was calibrated in wavelength using a mercury lamp with an accuracy of $\pm 0.1 \mathrm{~nm}$. The system was equipped with two photodetectors, specifically, a liquid nitrogen-cooled CCD (Roper instruments; LN/CCD400EB-GI) for measuring the spectra and a photomultiplier tube for capturing cathodoluminescence images. The acceleration voltages and current densities were $20 \mathrm{keV}$ and $10 \mathrm{nA}$, respectively. The spot size of the spectral measurements was 1 $\mu \mathrm{m}$.

\section{TGA}

The experiment was performed using an STA 2500 Regulus (Netzsch Japan, Ltd., Japan). The sample was placed on an $\mathrm{Al}_{2} \mathrm{O}_{3}$ holder with experiments conducted with heating at $10 \mathrm{~K} \mathrm{~s}^{-1}$ in a flow of Ar.

\section{AFM}

The experiment was performed on a MultiMode 8 (Bruker AXS, Ltd., USA) microscope (cantilever: RTESPA-150) operating under Ar in an MBRAUN glove box. AFM images were obtained by quantitative nanomechanical mapping mode. The height was calibrated using a commercial standard sample of Pt/glass (180 nm depth, VGRP-15M, Bruker AXS, Ltd., USA).

\section{EEM}

The experiment was performed using a spectrofluorophotometer (F-7000, Hitachi High-Tech Science Corporation, Ltd., Japan). For evaluation, BS samples were dispersed in acetonitrile, and their EEM spectra were recorded under liquid nitrogen temperature.

\section{UV-Vis}

UV-Vis absorption spectra of the powders were measured using a spectrophotometer (V-660, Jasco Ltd., Japan) with an integration sphere unit in diffuse reflectance mode. UV-Vis spectra of BS nanosheets dispersed in acetonitrile were measured under transmittance mode using a quartz cell.

\section{Photoelectrochemical properties}

r-BS electrodes were evaluated in an aqueous solution, in which $0.2 \mathrm{M}$ of sodium sulfate was dissolved at the $\mathrm{pH}$ of 7 . The r-BS or 2D BS nanosheets were deposited onto a fluorine-doped tin oxide coated substrate by a drop cast method using a solution dispersed in acetonitrile with Nafion. Photocurrent under rest potential was recorded using a potentiostat (HZ-5000, Hokuto Denko Corp). The light was irradiated using a $500 \mathrm{~W}$ xenon lamp passed through short-wavelength cutoff filters (cutoff under 350, 400, 422, and $610 \mathrm{~nm}$ ). The working, counter, and reference electrodes were r-BS film, platinum, and $\mathrm{Ag} / \mathrm{AgCl}$, respectively.

\section{Computational details}

Geometric and electronic structure calculations were performed within the framework of DFT using the Quantum ESPRESSO package. ${ }^{41,42}$ The LDA was used to describe the electron-electron 
interaction. ${ }^{43}$ All the computations were performed within DFTLDA framework, except for the estimation of the band gaps where the HSE06 hybrid functional method ${ }^{44}$ was used in addition to the LDA since LDA is known to underestimate the band gap values. The valence (B-2s, B-2p, S-3s, and S-3p) wavefunctions were expanded on a plane-wave basis up to a cutoff energy of $120 \mathrm{Ry}$. The core states were treated as normconserving pseudopotentials ${ }^{45,46}$ with $9 \times 9 \times 9$ and $9 \times 9 \times 1$ grids used for $k$-point sampling in the r-BS and BS nanosheets, respectively. The lattice parameters and the atomic positions were relaxed until the atomic forces were less than $10^{-4} \mathrm{Ry} / a_{0}$. The PBE generalized gradient approximation ${ }^{47}$ with semiempirical correction for van der Waals force ${ }^{48,49}$ was used during the structural optimization for HSE06 calculations. In the BS nanosheet calculations, the lattice dimension perpendicular to the sheet was fixed to $40 \AA$. The stacking pattern of the multilayer BS nanosheets were assumed to be the same with that of bulk r-BS (ABC stacking). By comparing DFT total energies, we confirmed that this is the most stable stacking in thin-layer BS nanosheets.

\section{Author contributions}

H. K., R. I., and M. T. contributed equally to this work. A. Y. conducted amorphous boron synthesis. H. K., R. I., M. Miyaka, and T. Ta conducted r-BS synthesis. H. K., R. I., K. H., and T. K. conducted XPS analysis. H. K., R. I., E. N., and T. K. conducted XRD analysis. E. N. conducted SRXRD measurements and Rietveld analysis. T. F., T. To, R. I., and T. K., conducted TEM, EELS, EDS, and electron diffraction analysis. H. K., R. I., L. L., S. L. S., and T. K. conducted SEM, EPMA, and EDX analysis. H. K., R. I., M. S. L. L., T. S., and T. K. conducted Raman scattering and Raman mapping analysis. H. K., R. I., K. Matsu, T. M., and T. K. conducted AFM analysis. H. K., R. I., and K. W. conducted CL analysis, H. K., and T. K. conducted TGA analysis. H. K., K. Miya, M. Miyau, and T. K. conducted EEM analysis. M. T., and S. S. conducted DFT band-structure analysis. S. L. S. fabricated BS electrodes, and K. Miya and M. Miyau investigated their photoelectrochemical properties. K. Miya, and M. Miyau conducted UV-Vis analysis. H. K., R. I., M. T., T. F., T. To, A. Y., M. Miyaka, K. Matsu, K. Miya, L. L., S. L. S., M. S. L. L., T. S., E. N., T. M., K. H., K. W., S. S., M. Miyau, T. Ta, H. H., and T. K. discussed the results. T. K. wrote the manuscript.

\section{Conflicts of interest}

There are no conflicts to declare.

\section{Acknowledgements}

We thank Prof. Y. Majima at Tokyo Tech., Dr G. Kobayashi at Institute for Molecular Science, Prof. J. Fujioka, Prof. T. Koyano, and Prof. H. Yanagihara of Univ. Tsukuba, and Dr N. Sato and Dr T. Mori at NIMS, for the discussion of the physical properties of r-BS. We also thank $\mathrm{Mr} \mathrm{Fu}$ Wei for the help in Raman mapping measurement. This work was supported by JSPS Kakenhi (Grant No. JP19H01823, JP19H02551, JP19H05046:A01,
JP21H00015:B01). This work was also financially supported by the MEXT Element Strategy Initiative to Form Core Research Center (JPMXP0112101001), Ogasawara Foundation for the Promotion of Science \& Engineering, and MHI Innovation Accelerator LLC. The synchrotron experiments were performed at SPring-8 BL02B2 with the approval of the Japan Synchrotron Radiation Research Institute (JASRI) as a Partner User (Proposal No. 2019A0068). We also thank the National Institute for Materials Science (NIMS) Battery Research Platform.

\section{References}

1 C. Tan, X. Cao, X.-J. J. Wu, Q. He, J. Yang, X. Zhang, J. Chen, W. Zhao, S. Han, G.-H. H. Nam, M. Sindoro and H. Zhang, Chem. Rev., 2017, 117, 6225-6331.

2 M. Osada and T. Sasaki, Adv. Mater., 2012, 24, 210-228.

3 D. Deng, K. S. Novoselov, Q. Fu, N. Zheng, Z. Tian and X. Bao, Nat. Nanotechnol., 2016, 11, 218-230.

4 A. K. Geim and I. V. Grigorieva, Nature, 2013, 499, 419-425. 5 Y. Liu, Y. Huang and X. Duan, Nature, 2019, 567, 323-333.

6 Y. Cao, V. Fatemi, S. Fang, K. Watanabe, T. Taniguchi, E. Kaxiras and P. Jarillo-Herrero, Nature, 2018, 556, 43-50.

7 Z. Zhang, E. S. Penev and B. I. Yakobson, Chem. Soc. Rev., 2017, 46, 6746-6763.

8 T. Kondo, Sci. Technol. Adv. Mater., 2017, 18, 780-804.

9 Y. Jiao, F. Ma, J. Bell, A. Bilic and A. Du, Angew. Chem., Int. Ed., 2016, 55, 10292-10295.

10 A. R. Oganov and V. L. Solozhenko, J. Superhard Mater., 2009, 31, 285-291.

11 I. Boustani, Surf. Sci., 1997, 370, 355-363.

12 E. S. Penev, S. Bhowmick, A. Sadrzadeh and B. I. Yakobson, Nano Lett., 2012, 12, 2441-2445.

$13 \mathrm{X} . \mathrm{Wu}, \mathrm{J}$. Dai, Y. Zhao, Z. Zhuo, J. Yang and X. C. Zeng, ACS Nano, 2012, 6, 7443-7453.

14 D. Fan, C. Yang, S. Lu and X. Hu, Appl. Phys. Lett., 2020, 117, 013103.

15 F. M. Arnold, G. Seifert and J. Kunstmann, J. Phys. Commun, 2020, 4, 031001.

16 Z. Zhu, X. Cai, C. Niu, C. Wang and Y. Jia, Appl. Phys. Lett., 2016, 109, 1-6.

17 A. J. Mannix, B. Kiraly, M. C. Hersam and N. P. Guisinger, Nat. Rev. Chem., 2017, 1, 0014.

18 S. Y. Xie, Y. Wang and X. Bin Li, Adv. Mater., 2019, 31, 1900392.

19 P. Ranjan, T. K. Sahu, R. Bhushan, S. S. R. K. C. Yamijala, D. J. Late, P. Kumar and A. Vinu, Adv. Mater., 2019, 31, 1-8.

20 H. Nishino, T. Fujita, N. T. Cuong, S. Tominaka, M. Miyauchi, S. Iimura, A. Hirata, N. Umezawa, S. Okada, E. Nishibori, A. Fujino, T. Fujimori, S. Ito, J. Nakamura, H. Hosono and T. Kondo, J. Am. Chem. Soc., 2017, 139, 13761-13769.

21 Q. Li, V. Surya, C. Kolluru, M. S. Rahn, E. Schwenker, S. Li, R. G. Hennig, P. Darancet, M. K. Y. Chan and M. C. Hersam, Science, 2021, 1148, 1143-1148.

22 C. Hou, G. Tai, J. Hao, L. Sheng, B. Liu and Z. Wu, Angew. Chem., 2020, 132, 10911-10917. 
23 S. Tominaka, R. Ishibiki, A. Fujino, K. Kawakami, K. Ohara, T. Masuda, I. Matsuda, H. Hosono and T. Kondo, Chem, 2019, 6, 1-13.

24 S. Demirci, N. Avazll, E. Durgun and S. Cahangirov, Phys. Rev. B, 2017, 95, 1-8.

25 P. Mishra, D. Singh, Y. Sonvane and R. Ahuja, Sustain. Energy Fuels, 2020, 4, 2363-2369.

26 P. Mishra, D. Singh, Y. Sonvane and R. Ahuja, J. Appl. Phys., 2020, 127, 184305.

27 T. Sasaki, H. Takizawa, K. Uheda and T. Endo, Phys. Status Solidi B, 2001, 223, 29-33.

28 K. A. Cherednichenko, I. A. Kruglov, A. R. Oganov, Y. Le Godec, M. Mezouar and V. L. Solozhenko, J. Appl. Phys., 2018, 123, 1-18.

29 K. A. Cherednichenko, P. S. Sokolov, A. Kalinko, Y. Le Godec, A. Polian, J. P. Itié and V. L. Solozhenko, J. Appl. Phys., 2015, 117, 185904.

30 Y. Shoji, T. Matsuo, D. Hashizume, H. Fueno, K. Tanaka and K. Tamao, J. Am. Chem. Soc., 2010, 132, 8258-8260.

31 Y. Wang, B. Quillian, P. Wei, C. S. Wannere, Y. Xie, R. B. King, H. F. Schaefer, P. V. R. Schleyer and G. H. Robinson, J. Am. Chem. Soc., 2007, 129, 12412-12413. 32 S.-D. Li, H.-J. Zhai and L.-S. Wang, J. Am. Chem. Soc., 2008, 130, 2573-2579.

33 A. D. Becke and K. E. Edgecombe, J. Chem. Phys., 1990, 92, 5397-5403.

34 F. Fuster, A. Sevin and B. Silvi, J. Phys. Chem. A, 2000, 104, $852-858$.

35 R. K. Defo, S. Fang, S. N. Shirodkar, G. A. Tritsaris, A. Dimoulas and E. Kaxiras, Phys. Rev. B, 2016, 94, 1-11.

36 K. F. Mak, C. Lee, J. Hone, J. Shan and T. F. Heinz, Phys. Rev. Lett., 2010, 105, 2-5.

37 S. Susarla, A. Kutana, J. A. Hachtel, V. Kochat, A. Apte, R. Vajtai, J. C. Idrobo, B. I. Yakobson, C. S. Tiwary and P. M. Ajayan, Adv. Mater., 2017, 29, 1-8.

38 A. K. Geim, Science, 2009, 324, 1530-1535.
39 S. Yamaoka, M. Akaishi, H. Kanda, S. Osawa, T. Taniguchi, H. Sei and J. Fukunaga, J. High Pressure Inst. Jpn., 1992, 30, 249-258.

40 E. Nishibori, E. Sunaoshi, A. Yoshida, S. Aoyagi, K. Kato, M. Takata and M. Sakata, Acta Crystallogr., Sect. A: Found. Crystallogr., 2007, 63, 43-52.

41 P. Giannozzi, S. Baroni, N. Bonini, M. Calandra, R. Car, C. Cavazzoni, D. Ceresoli, G. L. Chiarotti, M. Cococcioni, I. Dabo, A. Dal Corso, S. de Gironcoli, S. Fabris, G. Fratesi, R. Gebauer, U. Gerstmann, C. Gougoussis, A. Kokalj, M. Lazzeri, L. Martin-Samos, N. Marzari, F. Mauri, R. Mazzarello, S. Paolini, A. Pasquarello, L. Paulatto, C. Sbraccia, S. Scandolo, G. Sclauzero, A. P. Seitsonen, A. Smogunov, P. Umari and R. M. Wentzcovitch, J. Phys. Condens. Matter, 2009, 21, 395502.

42 P. Giannozzi, O. Andreussi, T. Brumme, O. Bunau, M. Buongiorno Nardelli, M. Calandra, R. Car, C. Cavazzoni, D. Ceresoli, M. Cococcioni, N. Colonna, I. Carnimeo, A. Dal Corso, S. de Gironcoli, P. Delugas, R. A. Di Stasio Jr, A. Ferretti and J. Flo, Phys. Condens. Matter, 2017, 29, 465901.

43 J. P. Perdew and A. Zunger, Phys. Rev. B: Condens. Matter Mater. Phys., 1981, 23, 5048-5079.

44 J. Heyd, G. E. Scuseria and M. Ernzerhof, J. Chem. Phys., 2006, 124, 219906.

45 S. Goedecker and M. Teter, Phys. Rev. B: Condens. Matter Mater. Phys., 1996, 54, 1703-1710.

46 C. Hartwigsen, S. Goedecker and J. Hutter, Phys. Rev. B: Condens. Matter Mater. Phys., 1998, 58, 3641-3662.

47 J. P. Perdew, K. Burke and M. Ernzerhof, Phys. Rev. Lett., 1996, 77, 3865.

48 S. Grimme, J. Comput. Chem., 2006, 27, 1787-1799.

49 A. V. Vincenzo Barone, M. Casarin, D. Forrer, M. Pavone and M. Sambi, J. Comput. Chem., 2009, 30, 934-939.

50 K. Momma and F. Izumi, J. Appl. Crystallogr., 2011, 44, 12721276. 